\title{
An Examination of Health Disparities in Louisiana Parishes Resulting from Clinic Closures and Policy Consolidation
}

\author{
Anita Feist \\ Department of Public Policy, Southern University and A \& M College, Baton Rouge, LA
}

\begin{abstract}
It has been observed in the healthcare and public policy literature that the healthcare market seems to be failing despite the tremendous efforts by policymakers, lawmakers, and different political leaders. In relation to the existing public health data, it is further observed in the literature that the current health care reforms, public health clinics closures and policy consolidations following the implementation of the affordable care act appear to be disappointing in the state of Louisiana - in relation to the high spread of STDs, breast-cancer, and low preventive screening participation across the parishes in Louisiana. As a result, this current study has utilized secondary data source to examine the health disparities in Louisiana state parishes resulting from the public health clinic closure and consolidation policies on all the preventative practices by utilizing data from Louisiana Department of Health. As part of the study design, the researcher utilizes descriptive design, bar chart and piechart to analyze the health disparities data following the clinic closures. The study suggested that the federal governments, state governors and local governments should reconsider the reopening of all the closures of the public health clinics in order to ensure a greater access to healthcare and healthy population among the Louisiana residents among the different parishes.
\end{abstract}

Keywords: Health, Clinic, Parishes, Policy, Healthcare, Screening, and STDs.

DOI: $10.7176 / \mathrm{JLPG} / 103-07$

Publication date: November $30^{\text {th }} 2020$

\section{INTRODUCTION}

In the literature of Harold Lasswell (1936) titled, "Power and Society: A Framework for Political Inquiry" defines politics as Who Gets What, When, How. It is important to note that the government actions and inactions do affect the lives of citizens. Surprisingly, many governments' efforts towards healthcare market seem to be failing. This is because the current health care reforms, public health clinics closures and policy consolidations following the implementation of the affordable care act appear to be disappointing in the state of Louisiana. Currently, many parishes, is battling with low health care professional's availability, and high prevalence of poor health outcomes (including high spread of STDs, high cancer spread, low accessibility to healthcare, and several communicable diseases) making all the public health interventions programs or initiatives looks bad in the face of the policy reforms. In fact, health disparities have become a major concern to policymakers and healthcare, especially in United States of America during this particular COVID-19 pandemic.

Interestingly, the World Health Organization (2010), defines health inequality/disparities as differences in health status and/or in the distribution of health determinants between different population groups. Also, scientists and policy-makers alike have recognized that health is affected by an assortment of factors that operate on multiple levels and throughout a person's lifetime (Adler \& Stewart, 2010). Although disparities in health status and health care have been readily researched, there is abundant evidence confirming that socioeconomic circumstances, as well as physical and social features of communities or neighborhoods, in tandem with education and work/work environments represent the root causes of health disparities and are associated with a vast number of health outcomes. Meanwhile, it has been observed in the literature that health informed policies and procedures are imperative in achieving health equity, and driving the reduction and eventual elimination of health disparities. If scientists, policy-makers, and communities integrate health factors in a systematic way by developing and implementing policies and programs in the midst of access to available health care facilities, the prevention of poor health increases while adverse health consequences decrease. However, it is not clear whether the policy consolidation and public health clinics closures influences the high spread of diseases and health disparities across the parishes. This particular study investigates this literature gap.

\section{LITERATURE}

Accessibility of Health Coverage on Income

Income inequality and poverty in the United States are high and are increasingly rising. There is also a link between poor health and poverty. According to Johns Hopkins Center for Health Equity, "low income is a major contributor to higher incidence and severity of illness and earlier deaths." The greater one's income, the less likely one will suffer from disease and premature death (National Center for Health Statistics, 2012). In 2009 it was determined that $45 \%$ of low-income adults under age 65 lack health insurance (Kaiser Family Foundation, 2009). Therefore, not only is higher income associated with better health and health outcomes, it is associated 
with one's accessibility to health coverage. According, to an analysis of data conducted by Lillian Thomas and Kevin Crowe of the Pittsburgh Post-Gazette and Milwaukee Journal Sentinel, respectively (2014) data from the largest United States metropolitan areas showed that residents in the poorest neighborhoods are less healthy and more likely to live in areas with physicians and hospital shortages. The analysis determined that an estimated two-thirds of hospitals that opened since 2000 are mostly in suburban areas.

At a time where hospitals and services are needed most for the less healthy in underserved and low-income communities, hospitals are closing their doors and doctors are following privately insured patients to more affluent areas. Moreover, according to a publication by the Kaiser Family Foundation (2016), the likelihood of being healthy decline with lower income

Income and wealth directly support better health and in contrast to those who earn low incomes, high income earners can afford the resources that protect and improve health. According to the Urban Institute and Virginia Commonwealth University's Center on Society and Health (2015) people with low incomes tend to have restricted access to medical are and are more likely to be uninsured or underinsured face barriers and other health related expenses. Pre-implementation of the Affordable Care Act, in 2010, the probability of being uninsured before the age of sixty-five is 28 percent for those living in poverty, 16.5 percent for those with incomes two to three times the federal poverty level (FPL), and 5.2 percent for those with incomes four or more times the FPL (National Center for Health Statistics, 2014).

Figure 1: Estimated Uninsured Rates by Income

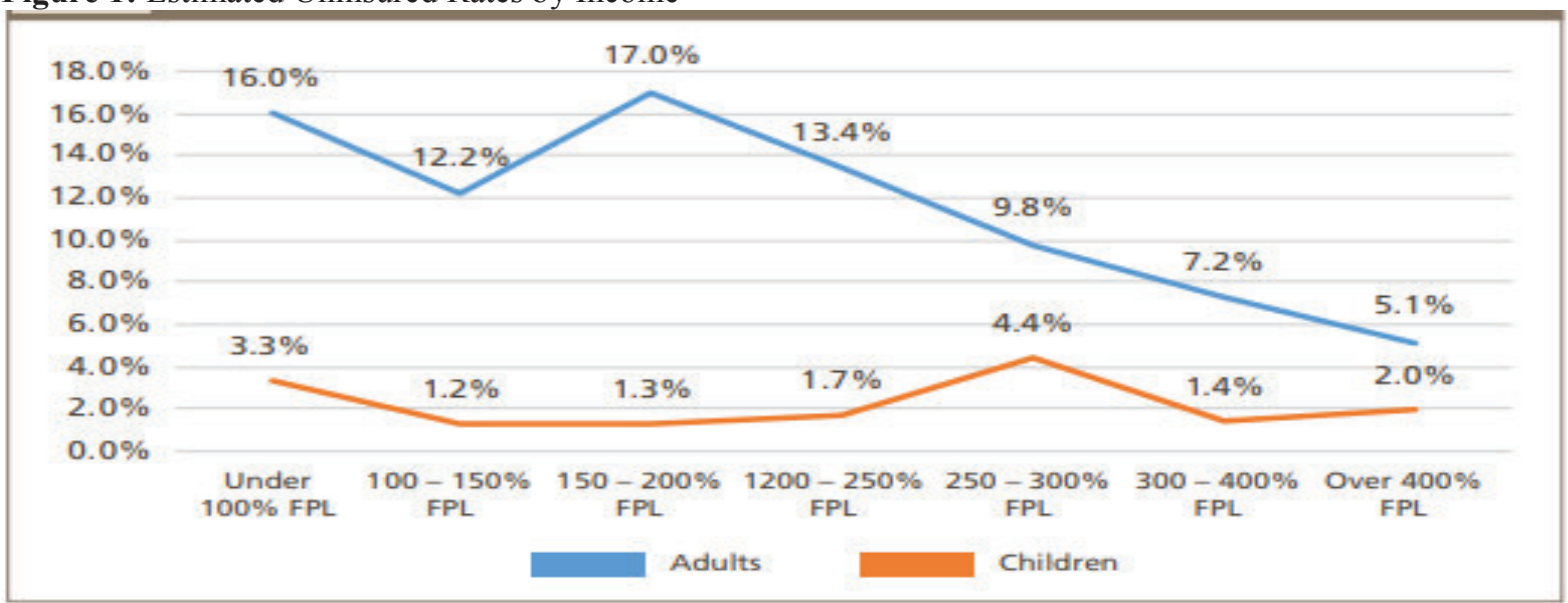

Source: Louisiana Health Insurance Survey, 2017

With the income of women continuously lagging that of their male counterparts, coupled with the rising costs of health insurance and health care, many women are foregoing treatment and care. In 2010, it was reported that an estimated twenty-six million women (approximately thirty-three percent) exhausted ten $\%$ or more of their income on premiums and out of pocket expenses for themselves and their families (see Figure 1 for more details). This twenty-five percent (25\%) rise from 2001 has left many working women struggling with the decision to put the health care needs of their families over their own (Schoen, Stremikis, How, and Collins, 2010).

\section{Accessibility of Health Coverage on Region}

According to the Organization for Economic Cooperation and Development

(OECD) (2014) health care use not only varies across countries but within countries as well. Author of The Constitution of Society, Anthony Giddens writes, it is the physical description of boundaries that determines how we view communities and how societies are constructed. How health services are structured, how and when people use such services, and their health status are factors that can be determined by their geography and in turn create disparities.

Across states and broader, regions, there are disparities in both the availability and costs of health insurance coverage. For example, the Kaiser Family Foundation reported in their "Employer Health Benefits Survey" (2015) that while the average single and family premiums for those employees who are covered are higher in the Northeast and lower in the South than the average premiums for those who are covered in other regions, in the Southern region employees are less likely to provide coverage for an employee's domestic partner than other regions. It was reported in the South $41 \%$ of employers provide benefits for same-sex partners compared to $51 \%$ in the Northeast.

It should also be noted that there are factors as to why health care costs vary significantly by region. These are variations in cost of services between regions; provider competition or lack thereof; health plan competition or lack thereof; and prevalence of rural communities (Riley \& Cousart, 2017). When there is a lack of provider 
competition and a lack of health plan competition in regions it not only allows providers to set rates for services, but in areas where there is less enrollment and less interest by health plans to participate in the market there is less completion among health plans which in turn leads to higher rates leading to higher premiums. Furthermore, rural communities encompass a combination of the factors lending to less choice and higher premiums (Riley \& Cousart, 2017).

\section{Accessibility of Health Coverage on Gender}

As stated previously, studies show that for women, the statistics are more daunting. Studies from the National Health Interview Survey (NHIS) provided evidentiary support of disparities in healthcare and health outcomes between men and women (Fraizer, 2009). According to the American Heart Association (2013), one in five women between the ages of 18 and 64 are uninsured with a higher probability of women receiving inadequate access to care with poorer health outcomes. Furthermore, the Kaiser Family Foundation (2010) believes women are falling through the cracks of an elusive healthcare system and while the foundation acknowledges the need for quality care of both sexes, they advocate that women have more complex needs including pregnancy, related reproductive health needs, and higher incidence of chronic illness. Moreover, women tend to live longer in the United States (Braws,1997) and more often than not use more health care services throughout their lives.

According to the National Institute of Health Report (1999), two-thirds of all inpatient, outpatient, and pharmaceutical services are used by women. When compared to men, women are also more susceptible to immunological, neurologic, and psychiatric disorders; as well as disorders that are often associated with poverty or violence (Wentz, 1995). The Society for the Advancement of Women's Health Research (1998) has also reported that women also suffer from higher rates of cardiovascular disease once they reach the age of 65 . It is also important that women's health care is addressed as it has been shown that women are the major decision makers on health issues for their family (Nussbaum, 2000). Therefore, by coordinating healthcare for themselves and for their families, women consume two-thirds of all medical care (Braus, 1997).

Although women consume the majority of health services, according to a poll conducted by The New York Times/CBS News Poll regarding women's health issues, Americans believe that the federal government spends more money researching the effect of diseases have on men than the effect they have on women (Elder, 1997). While women make up over half of the population in the United States (United States Census Bureau, 2012) their health needs are not equally researched and are lagging when compared to that of men. It was concluded by the Public Health Service Task Force on Women's Health Issues in 1985 that "the historical lack of research focus on women's health concerns has compromised the quality of health information available to women as well as the healthcare they receive."

Thirty-two years after the Public Health Service Task Force's findings, women are still seeking adequate research and quality care to meet the needs of their families as well as themselves. The need for quality, affordable and accessible health services has long been an issue for women in the United States. With the formation of the Women's Health Movement in the late 1960s it was realized that the current system of health care did not adequately address the needs of women. While gains in women's health research have been made, continuous efforts regarding not only the reproductive needs of women but comprehensive healthcare needs must be addressed. Such research includes, but is not limited to, sexual and reproductive health; mental health; health disparities based on gender, race, and ethnicity; as well as accessibility to and affordability of health services.

According to the Kaiser Family Foundation's Women's Health Care Chartbook (2011), in 2007 one in four women nationwide reported having to delay or go without preventive care due to costs of services. Within two years more than half of American women went without preventive services (Whitehouse.gov/health reform, 2012). When women do not have access to preventive care they become at risk for poorer health while increasing their chances of acquiring chronic health conditions (Kaiser Family Foundation, 2011). In relation to Figure 2, despite the United States being the most industrialized nation, women, whether insured or uninsured, are more likely to encounter problems in accessing needed health services when compared to women in countries where universal health coverage exist (Robertson, Squires, Garber, Collins, \& Doty, 2012). 


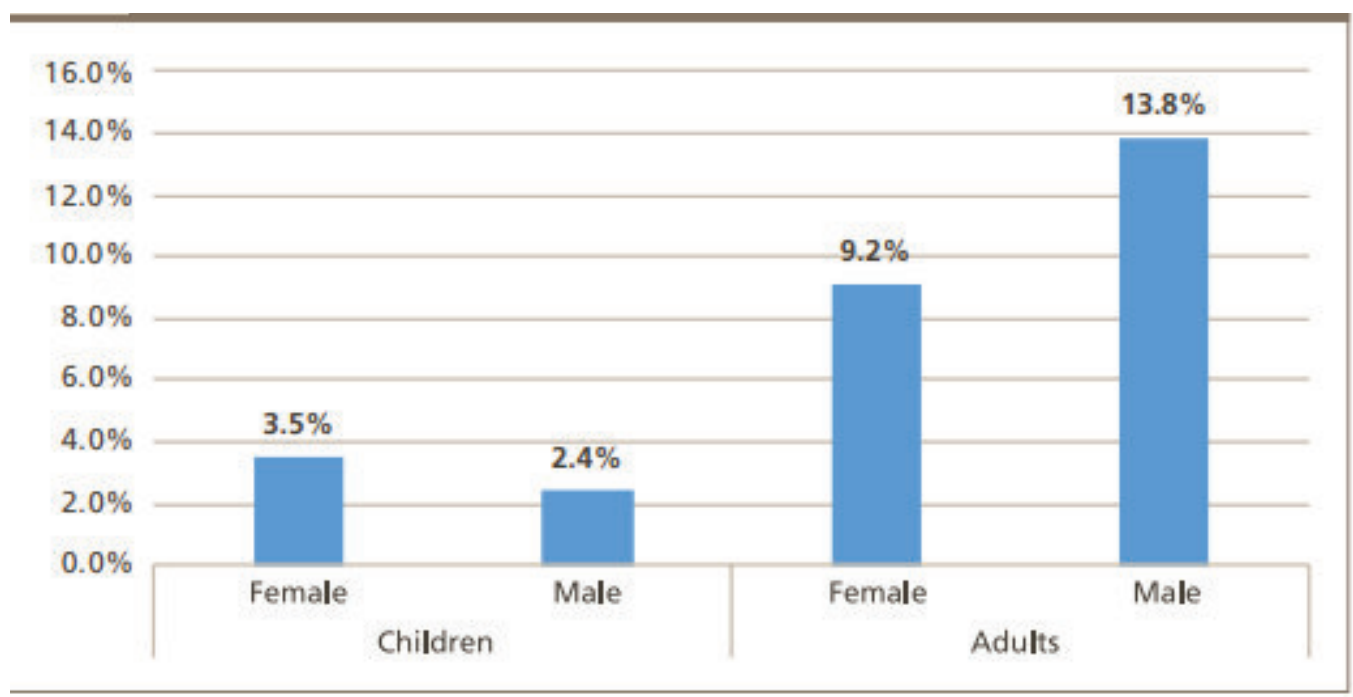

Figure 2: Uninsured Rates by Gender

Source: Kaiser Family Foundation, 2011

Accessibility of Health Coverage on Age

The United States Census Bureau (2014) noted that working age adults were among those with the highest uninsured rates. According to the Kaiser Commission on Medicaid and the Uninsured (2014), over 48 million non-elderly Americans did not possess health insurance in 2011. Additionally, according to Jessica Barnett and Marina Vornovitsky (2016) with the United States Census Bureau, "age is strongly associated with the likelihood that a person has health insurance and the type of health insurance a person has." Researchers concluded that this segment of the population constructed a disproportionate share of the uninsured due to the inability to obtain Medicaid with only $17.6 \%$ of the non-elderly population covered by Medicaid and SCHIP.

Before the passing of the Patient Protection and Affordable Care Act (ACA) in 2010, insurance rates were on a decline for children but increasing for nonelderly adults. This decline in rates for children could be attributed to increases in public coverage through both Medicaid and the Children's Health Insurance Program (CHIP) (Rosenbaum and Kenney, 2014). The passage of CHIP in 1997 increased access to public coverage through the expansion of eligibility and programmatic changes with the goal of increasing enrollment of the nation's eligible children. Furthermore, in 2009 CHIP was reauthorized with increased eligibility expansions for children from low- and moderate-income families (Harrington, Kenney, et al., 2014).

For the near-elderly the outcome can be just as dire. The uninsured near-elderly represent a vulnerable population (Davis, 1997) where the consequence of not having health insurance is more severe (Johnson \& Crystal, 1997). As this segment of the population face higher premiums when acquiring health insurance, many tend to go without such coverage for longer periods putting their health in even greater risk for negative outcomes (Brennan, 2000). According to the authors of Health Insurance and Mortality: Evidence from a National Cohort (1993), there are increased mortality rates among uninsured near-elderly adults when compared to their insured peers. Studies have shown that adults age 51-61 years old who lack health insurance coverage experience an increased rate of decline in their overall health and physical functioning (Sudano \& Baker, 2006).

According to Lisa Yagoda (2005), author of "Older Adults and Health Disparities: The Impact on Access to Care," one-fourth of adults age 65 and older do not possess supplemental insurance coverage for their healthcare needs with $93.6 \%$ qualifying for Medicare. With the aging population (those over 65) contributing to the most to overall healthcare spending (Stanton, 2006) it is imperative to recognize this population's impact on the state of healthcare in the United States (see Figure 3 for more details). 


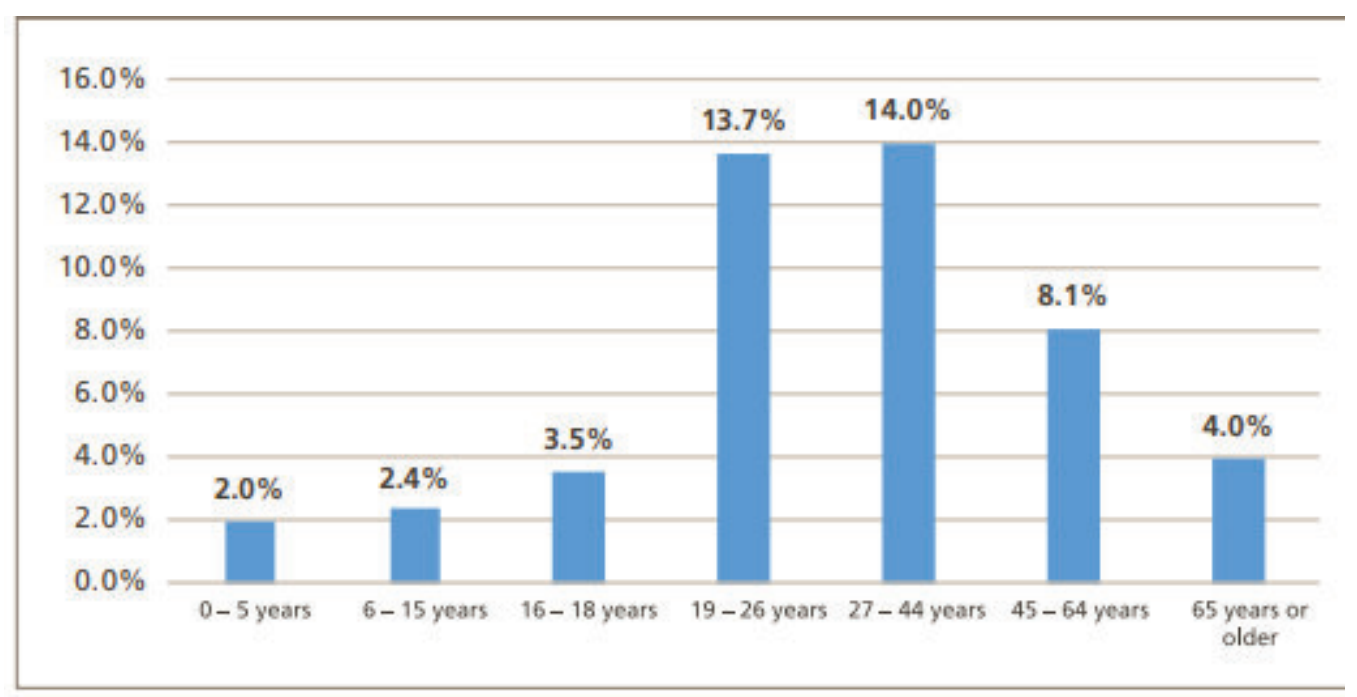

Figure 3: Uninsured Rates by Age

Source: Kaiser Family Foundation, 2011

Since the state's Medicaid expansion, the 2017 the expansion came at an opportune time to help buffer residents from the economic downturn in oil and gas with some of the most impacted regions still exhibiting the lowest uninsured rates. The survey demonstrated that uninsured rates among children remained very low at $2.4 \%$, which is in line with recent uninsured rates in Louisiana over the last several LHIS surveys. The most common type of coverage among children is Medicaid, with $53.9 \%$ of children covered by the program, which has helped keep uninsured rates low despite economic fluctuations over time. While employer coverage continues to be the leading source of coverage among adults, Medicaid is now playing an increasingly important role in providing coverage for adults and is the second most common type of coverage with $20.8 \%$ of adults covered by the program (see Figure 3 for more details).

\section{Health /Health Care Disparities}

$\begin{gathered}\begin{array}{c}\text { Access to Health } \\ \text { Coverage }\end{array} \\ \text { Care }\end{gathered} \mid \begin{gathered}\text { Quality of } \\ \text { Care }\end{gathered}$

The term disparity is often used as it relation to gender. However, disparities have the ability to be intersectional and can exist across dimensions, such as age, socioeconomic status, and geographical locations (Healthy People, 2016). Health disparities stem from health inequities - "differences in health status or in the distribution of health determinants between different population groups" (Barnes et al., 2016). It is important to understand the disparities present within the United States and the populations affected. In 2008, the population of the United States was an estimated at nearly 304 million people according to the (United States Census Bureau, 2008). An estimated $33 \%$ more than 100 million people identified themselves as a racial/ethnic minority (United States Census Bureau, 2008). Moreover, an estimated $23 \%$ of the population, 70 million people lived in rural areas of the country, while approximately $77 \%$ of the population 233 million people lived in urban areas (United States Census Bureau, 2008). In 2002, approximately 4 percent of the population, ages 18 to 44, identified themselves as gay, lesbian, bisexual, or transgender (Mayer, Bradford, Makadon, Stall, Goldhammer, \& Landers, 2008).

According to the Center for Disease Control's Health Disparities and Inequalities Report-United States, (2011), while the United States has made progress in improving health outcomes and in reducing disparities, ongoing economic, and gender disparities in health care still exist. A disparity refers to differences in the quality of health care across various population groups that can occur by gender, race and ethnicity, education level, income level, disability, or geographic location and can exist among all age groups including children, adolescents, and the near-elderly (Hebert, Sisk, \& Howell, 2008). These disparities in healthcare are forms of inequality. In fact, more often than not, low-income and ethnic minorities have a poor health status. It is important to analyze and study such causes of health disparities so that stake holders, policy makers, and healthcare professionals are informed regarding why disparities occur and thus take future decisions that may reduce these disparities.

Health care treatments and outcomes are influenced by one's ability to access health care and as researched by the Kaiser Family Foundation (2015) access to health care is associated with whether or not one is insured. In 2013, 41.3 million nonelderly Americans in the United States were without health insurance (Kaiser Family 
Foundation, 2014). The uninsured also have a higher mortality rate than those with private health insurance (Franks, Clancy, and Gold, 1993).

According to a study conducted by researchers at Harvard University and published in the November 2009 issue of Archives of Surgery, uninsured patients face health related disparities in health screenings, hospital admission, treatment, and outcomes when compared to insured patients. According to a Virginia study, patients insured are more likely to report being screened for colorectal cancer as uninsured equivalents of comparable ages (deBosset, Atashili, Miller, \& Pignone, 2008). Uninsured adults receive fewer preventive services including screenings for types of cancer as well as testing for high blood pressure and cholesterol. In fact, cancer rates for uninsured individuals are more likely to be diagnosed at a later stage of illness because treatment is unsuccessful. This ultimately increases mortality rates among the uninsured.

Louisiana is a state in the Deep South region of the South Central United States. It is the 19th-smallest by area and the 25th most populous of the 50 U.S. states. Louisiana is bordered by the state of Texas to the west, Arkansas to the north, Mississippi to the east, and the Gulf of Mexico to the south (see Figure 4 for more details). A large part of its eastern boundary is demarcated by the Mississippi River. Louisiana is the only U.S. state with political subdivisions termed parishes, which are equivalent to counties. The state's capital is Baton Rouge, and its largest city is New Orleans. Much of the state's lands were formed from sediment washed down the Mississippi River, leaving enormous deltas and vast areas of coastal marsh and swamp (Federal Reserve Bank of St. Louis, 2019). These contain a rich southern biota; typical examples include birds such as ibis and egrets. There are also many species of tree frogs, and fish such as sturgeon and paddlefish (Valdman, and Rottet, 2009). In more elevated areas, fire is a natural process in the landscape and has produced extensive areas of longleaf pine forest and wet savannas. These support an exceptionally large number of plant species, including many species of terrestrial orchids and carnivorous plants. Louisiana has more Native American tribes than any other southern state, including four that are federally recognized, ten that are state recognized, and four that have not received recognition (Dayna, 2013).

\section{Discussing Louisiana In the Context}

Some Louisiana urban environments have a multicultural, multilingual heritage, being so strongly influenced by a mixture of $18^{\text {th }}$ century French, Italian, Haitian, Spanish, Native American, and African cultures that they are considered to be exceptional in the U.S. Before the American purchase of the territory in 1803, the present-day State of Louisiana had been both a French colony and for a brief period a Spanish one (Murphy, 2008; U.S. Census Bureau, 2012). In addition, colonists imported numerous African people as slaves in the 18th century. Many came from peoples of the same region of West Africa, thus concentrating their culture. In the post-Civil War environment, Anglo-Americans increased the pressure for Anglicization, and in 1921, English was for a time made the sole language of instruction in Louisiana schools before a policy of multilingualism was revived in 1974 (Wayback Machine, 2016; Murphy, 2008). There has never been an official language in Louisiana, and the state constitution enumerates "the right of the people to preserve, foster, and promote their respective historic, linguistic, and cultural origins"(Wayback Machine, 2016; Murphy, 2008). Like other states in the Deep South region, Louisiana frequently ranks low in terms of health, education, and development, and high in measures of poverty (Wayback Machine, 2016; Murphy, 2008; U.S. Census Bureau, 2012). In 2018, Louisiana was ranked as the least healthy state in the country, with high levels of drug-related deaths and excessive alcohol consumption, while it has had the highest homicide rate in the United States since at least the 1990s (U.S. Census Bureau, 2012). 


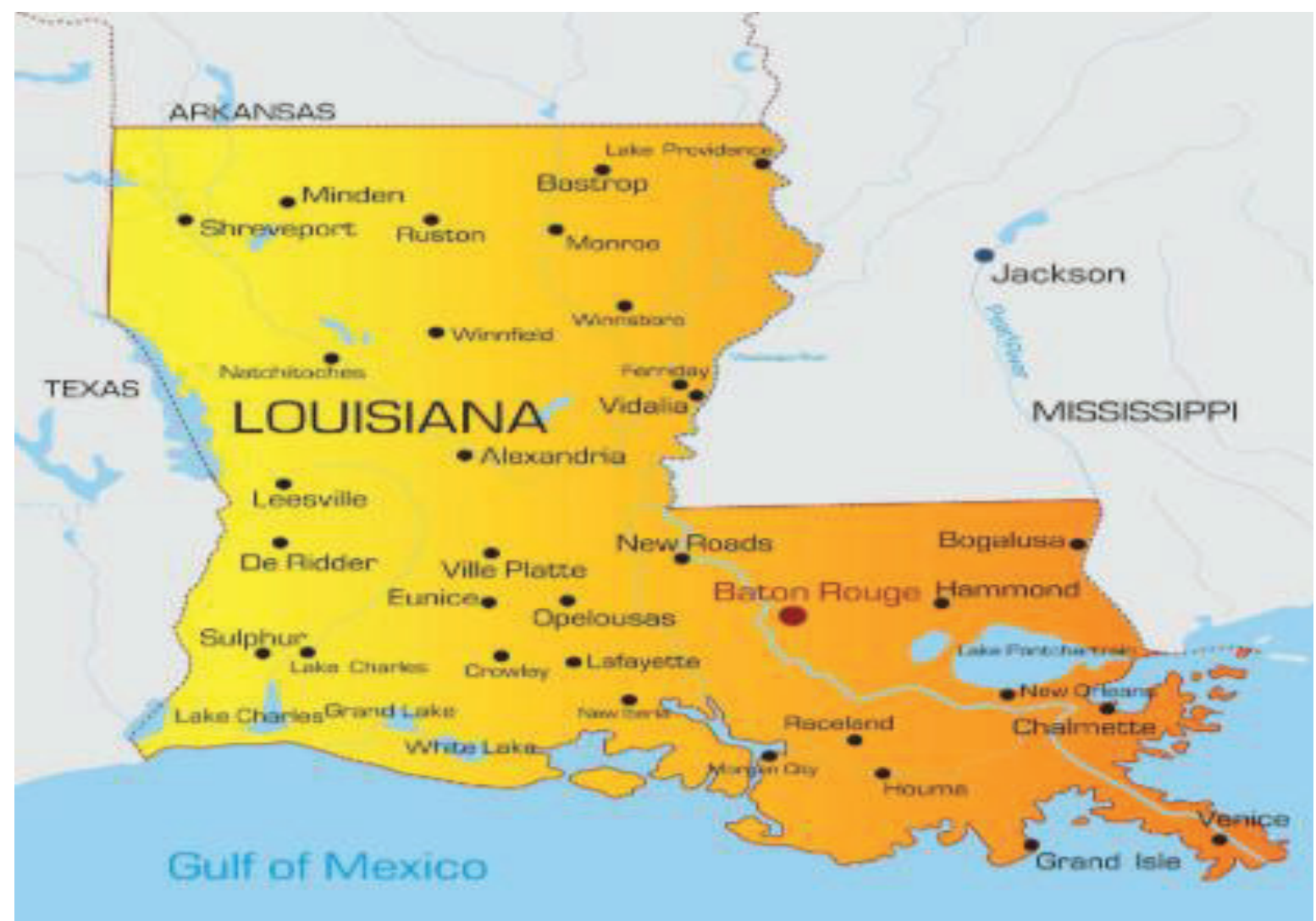

Figure 4: Political Map of Louisiana

Source: Author's Modification of Louisiana Geographical Map

\section{METHODS AND MATERIALS}

The study adopts a descriptive type of study. A secondary data was retrieved from the Louisiana Department of Health, and the Integrated Public Use Microdata Series USA (IPUMS-USA) which is the largest population database containing Microdata samples from the United States. It is observed in the literature that the IPUMS is housed at the Minnesota Population Center and provides individual level data on United States citizens from every United States Census since 1850, the American Community Survey since 2000, and the Content Population Survey since 1962. Data was also gathered from the Behavioral Risk Factor Surveillance System (BRFSS) which is composed of health-related telephone surveys that collect state-level data from U.S. residents regarding health-related risk behaviors, use of preventative services, and chronic health conditions. The BRFSS is a coordinated effort by the CDC and is administered annually using standard sets of core questions, modular questions that are state-optional, and customized questions drafted for the state's individual needs to overcome geographical health-related issues. Furthermore, data from the Louisiana Department of Health was used from a compilation of extracts from state and national databases that feature the last complete year of data available. The compilation of data extracts used in the data collection includes the Louisiana Department of Health Office of Behavioral Health and Office of Public Health Bureau of Infectious Diseases, and the STD/HIV program. All the data variables were measured on interval-ratio scale. Tables, bar graphs, and pie-charts were used to analyze the study.

\section{DATA ANALYSIS AND DISCUSSION}

This section of the data analysis represents the dynamics in STD infection rates across the parishes in Louisiana after the closure of public health clinics and policies consolidations starting from 2014/2015 fiscal year. The section discusses regional Chlamydia infection rates, Gonorrhea infections, and Syphilis infection rates in details. 


\section{Graphical Analyses of STD Prevention Practices across the Regions and Parishes in Louisiana.}

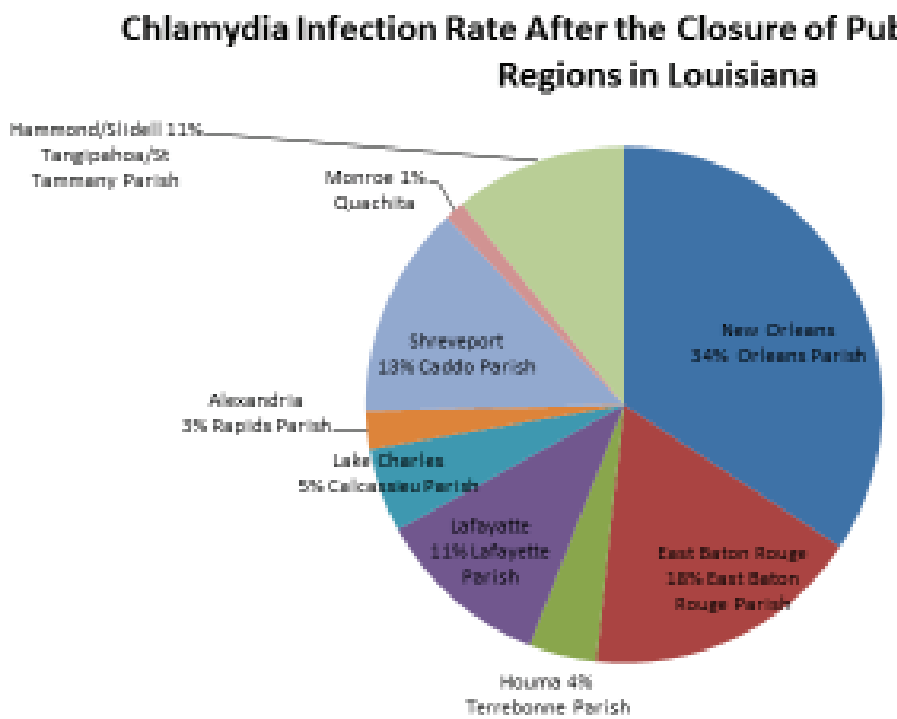

Figure 5: Chlamydia Infection Rate after the Closure of Public Health Clinic by Regions in Louisiana [2015/2016-2019]

Source of Data: Integrated Public Use Microdata Series USA (IPUMS-USA), 2020

Figure 5 discusses the Chlamydia infection rate after the closure of public health clinic by regions in Louisiana. Figure 5 reveals that New Orleans parishes/region was highly affected by the closures of the public health clinic by recording a very high Chlamydia infection rate of $34 \%$, followed by East Baton Rouge with $18 \%$, Shreveport with 13\%, Lafayette and Hammond/Slidell with 11\% each, Lake Charles with 5\%, Alexandria with $3 \%$ and Monroe with 1\% Chlamydia infection rates. This implies that the regional hotspot zone of Chlamydia infections based on the infection rate could include New Orleans, East Baton Rouge, Shreveport, Lafayette and Hammond/Slidell, since they all have infections rate more than 10\% (see Figure 5).

\section{Number of Gonorrhea Infections Patients after the Closure of Public Health Clinic by Regions in Louisiana}

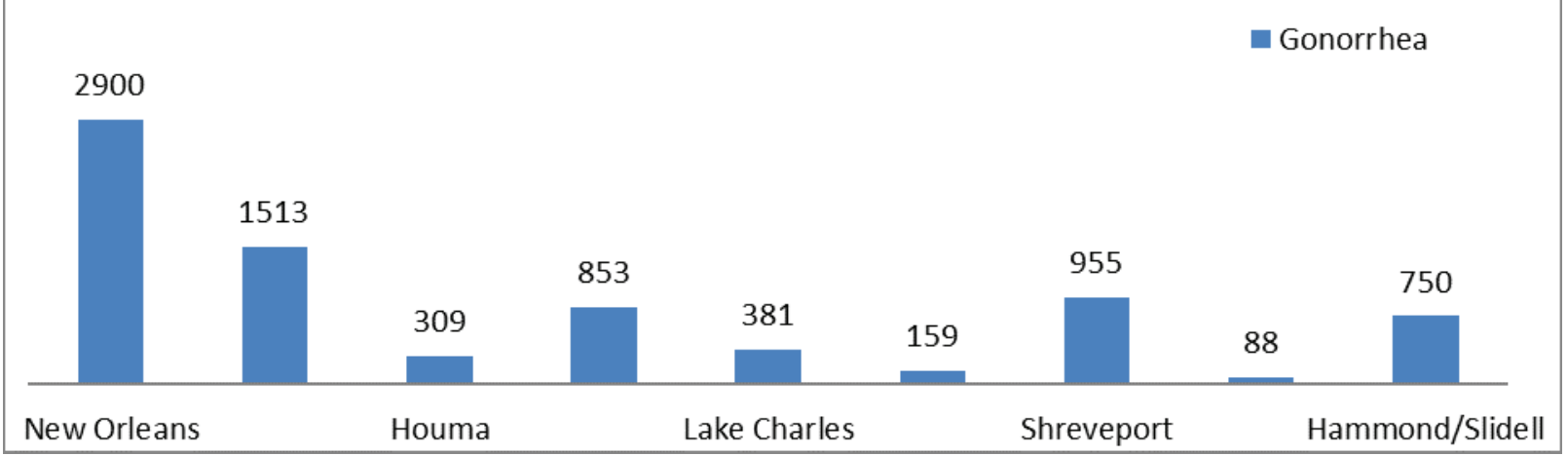

Figure 6: Number of Gonorrhea Infections Patients after the Closure of Public Health Clinic by Regions in Louisiana [2015/2016-2019]

Source of Data: Integrated Public Use Microdata Series USA (IPUMS-USA), 2020 
Figure 6 discusses the number of Gonorrhea infections patients after the closure of public health clinic by regions in Louisiana. Figure 6 reveals that New Orleans parishes/region recorded a very high number of Gonorrhea infections patients with an average regional value of 2,900 infection cases, followed by East Baton Rouge with 1,513 infection cases, Shreveport with 955 infection cases, Lafayette with 853 infection cases, Hammond/Slidell with 750 infection cases, Lake Charles with 381 infection cases, Houma with 309 infection cases, Alexandria with 159 infection cases and Monroe with 88 infection cases of the number of Gonorrhea infections patients. This implies that the regional hotspot zone of the number of Gonorrhea infections patients based on the number of infection cases could include only New Orleans, and East Baton Rouge, since they all have number of infections cases more than 1,000 (see Figure 4.2).

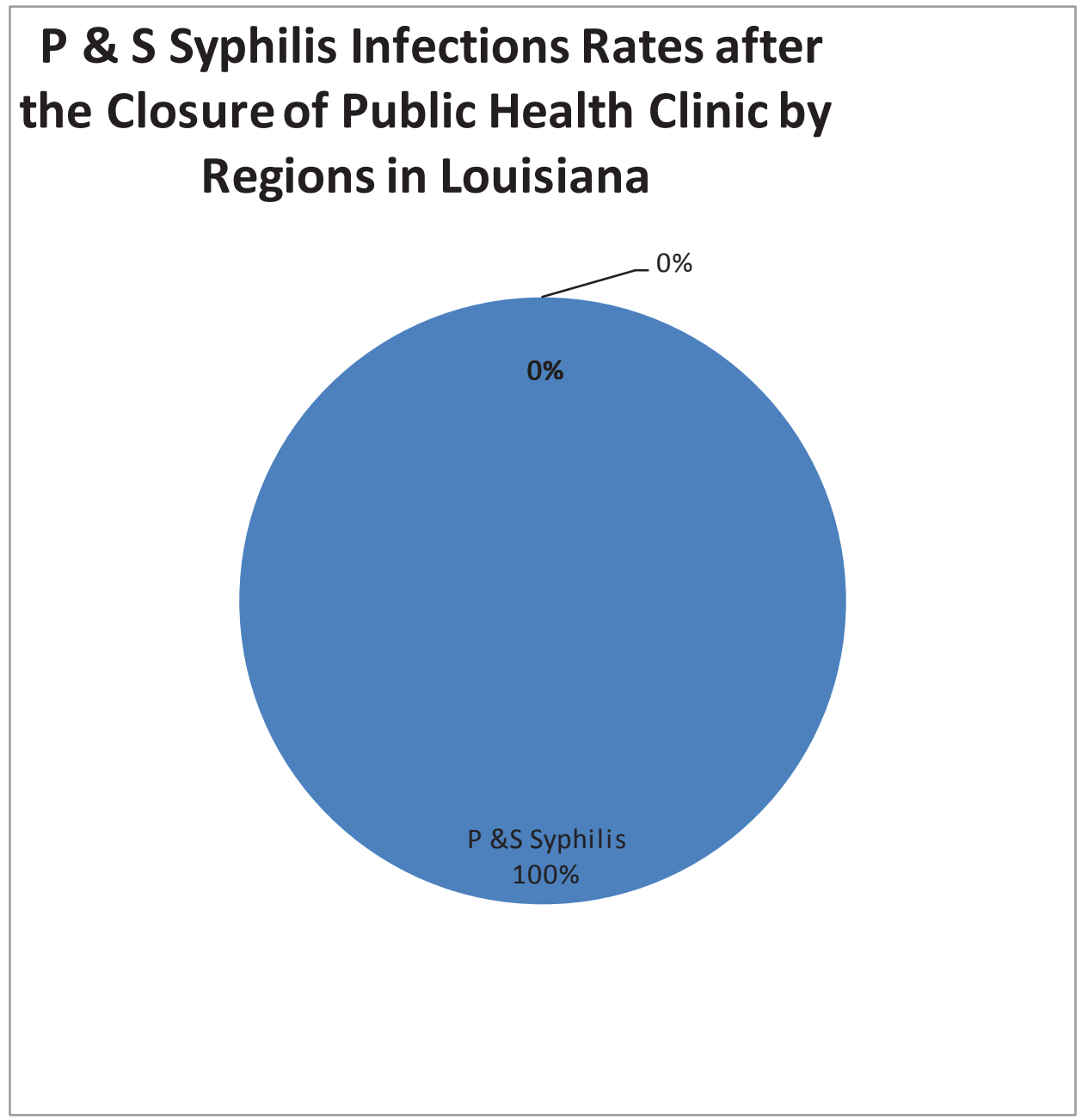

Figure 7: P \& S Syphilis Infections Rates after the Closure of Public Health Clinic by Regions in Louisiana [2015/2016-2019]

Source of Data: Integrated Public Use Microdata Series USA (IPUMS-USA), 2020

Figure 7 discusses the Primary (P) and Secondary (S) Syphilis infection rate after the closure of public health clinic by regions in Louisiana. Figure 7 reveals that New Orleans parishes/region was highly affected by the closures of the public health clinic by recording a very high P\&S Syphilis infection rate of 38\%, followed by Shreveport with 24\%, East Baton Rouge with 19\%, Lafayette with $8 \%$, Houma with 4\%, Lake Charles and Hammond/Slidell with 3\% each, Monroe with 1\%, and Alexandria with $0 \%$ of P\&S Syphilis infection rates. This implies that the regional hotspot zone of P\&S Syphilis infection based on the infection rate could include New Orleans, Shreveport, and East Baton Rouge, since they all have infections rate more than 10\% (see Figure 7 for more details).

\section{CONCLUSION AND POLICY IMPLICATIONS}

In conclusion, the analysis of the study reveals that the regional hotspot zone of Chlamydia infections based on the infection rate could include New Orleans, East Baton Rouge, Shreveport, Lafayette and Hammond/Slidell, since they all have infections rate more than $10 \%$ (see Figure 5 for more details). The study further reveals that the regional hotspot zone of the number of Gonorrhea infections patients based on the number of infection cases 
could include only New Orleans, and East Baton Rouge, since they all have number of infections cases more than 1,000 (see Figure 6 for details). Again, it was further observed from the data analysis that that the regional hotspot zone of P\&S Syphilis infection based on the infection rate could include New Orleans, Shreveport, and East Baton Rouge, since they all have infections rate more than 10\% (see Figure 7 for more details). Moreover, the study finds that the average Gonorrhea infection rate, Chlamydia infection rate, and P\&S Syphilis infection rate between 2014 and 2019 is greater than the average infection rate before the closure of the public health clinics (2008 and 2013).

\section{REFERENCES}

Birkland, T. (2011). An Introduction to the Policy Process: theories, concepts, and models of public policy making. $3^{\text {rd }}$ edition. Routledge, Taylor \& Francis Group.

Buchmuelier, J.W. (2006). How far to the hospital? The effect of hospital closures on access to care. Journal of Health Economics.

Cantor, B.S. (2010). Aiming Higher: Results from a State Scorecard on Health Systems Performance.

CDC MMWR (1995) Impact of Closure of Sexually Transmitted Disease Clinic on Public Health Surveillance of Sexually Transmitted Diseases. DC.

Center for Medicare and Medicaid Services, Office of the Actuary, National Health Statistics Group. (2010). Healthcare Expenditure Analysis. National Health Care Expenditure Data.

Centers for Medicare and Medicare Services. (2012). Medicare and Medicare Services. CMS. Retrieved from Centers for Medicare and Medicare Services.

Cunningham, N.B. (2006). Results from a State Scorecard on Health Systems Performance. NASHP

Currie, R. (1998) Distance to Hospitals and Children's Access to Care: Is Being Close Better, and for Whom? The National Bureau of Economic Research.

DC Goodman, E.F. (1997). The distance of community medical care and the likelihood of hospitalization: is loser always better? American Journal of Public Health, Vol. 87, Issue 7 1144-1150.

Deal LW, S.P. (1998). Medicaid managed care and children: an overview. Future Child, 93-104. DHH (2005). Making Medicaid Better FAQ's'.

DHH (2015/2016). Louisiana Annual Medicaid Report.

Eddy. (1997). Balancing cost and quality in fee for service versus managed care. Health Affairs, 162-173.

Grubar, J. (2016). Public Finance and Public Policy. 5 $^{\text {th }}$ edition. Worth Publishers: A Macmillan Education Imprint New York, pp. 3-23.

Havlovic, B.V. (1998). Coping with Downsizing and Job Loss. Canadian Journal of Administrative Science, $322-332$

Henry J. Kaiser-Family Foundation. (2010). Focus on Health Reform: Health Reform Implementation Timeline.

Hadley, J. (2003). Sicker and poorer: The consequences of being uninsured. Medical Care Research and Review, 60 (2, Suppl): 3S-75S. doi:10.1177/1077558703254101

Institute of Medicine. (2009). America's uninsured crisis: Consequences for health and health care. Washington, $D C$ : National Academies Press.

Isaac, S., \& Michael, W. B. (1981). Handbook in research and evaluation (2nd ed.). San Diego, CA: EDITS.

Jonk, Y. C.; Call, K. T., Cutting; A. H., O'Conner; H. Bansiya, V.; \& Harrison, K. (2005). Health care coverage and access to care: The status of Minnesota's veterans. Medical Care, 43(8), 769-774. Retrieved from http://www.jstor.org/stable/3768294

Jlida C.G.; Hyndman, C.D. (2000). Differential effects on socioeconomic groups of modeling the location of mammography screening clinics using Geographic Information Systems. Australian and New Zealand Journal of Public Health, volume 24, issue 3, pages 281-286.

Kaiser Family Foundation and Health Research and Educational Trust. (2009). Employer Health Benefits 2009 Annual Survey.

Kaiser Commission on Medicaid and the Uninsured. (2012). The Uninsured: A primer: Key facts About Americans without health insurance. Retrieved from https://kaiserfamilyfoundation.files.wordpress.com/2013/01/7451-08.pdf

Kaiser Family Foundation. (2008). Eliminating racial/ethnic disparities in health care: What are the options? Retrieved August, 2017, from 152 https:/www.kff.org/disparities-policy/issue-brief/eliminatingracialethnicdisparities-in-health-care-what/

Kaiser Family Foundation. (2009). Low-income adults under age 65-many are poor, Sick, and uninsured. Policy Brief, Publication \#7914 Retrieved from http://www.kff.org/healthreform/upload/7914.pdf

Kaiser Family Foundation. (2011). Health insurance coverage of women 19-64, states (2010-2011). Retrieved from http://www.kff.org/other/state-indicator/nonelderlyadult-women/

Kaiser Family Foundation. (2011). The uninsured: A primer, key facts about Americans without health insurance. Washington, DC: The Kaiser Commission on Medicaid and the Uninsured. 
Kaluzny, H.A. (1983). Hospital Closure: A review of Current Proposed Research. Health Services Research.

LA Department of Hospitals Bureau of Health Services Financing. (2010). Declaration of Emergency. LA Department of Hospitals Bureau of Health Services Financing.

Laswell, H. (1936). Politics: Who Gets What, When, How. Britannica. https://www.britannica.com/topic/Politics-Who-Gets-What-When-How

Larson, J.S. (1980). Why Government Programs Fail: Improving Policy Implementation, Greenwood Pub Group. Lindrooth, S.A. (2003). The Effect of Urban Hospital closure on Markets. Journal of Health Economics, 691712.

Los Angeles Department of Health Services Acute Communicable Disease Control. (1995). Clinic Closure Impact: Cross-Sectional Survey Report. Los Angeles.

Louisiana State University Public Policy Research Lab. (2009). Health Insurance Survey. Louisiana State University Public Policy Research Lab Baton Rouge.

Marmor T,O.J. (1998). Rethinking Medicare Reform. Health Affairs, 52-60.

Mayer, K.S.(1987). Patterns of Rural Hospital Closure in the United States. Social Science \& Medicine, 327-334.

McDonald, M., \& Hertz, R. (2008). A profile of uninsured persons in the United States. New York, NY: Pfizer.

McKay, C. (1995). Access implications of Rural Hospital Closures and Conversions. Hospital Service Administration, 227-246.

M.E. Sharpe. Patient Protection and Affordable Care Act, Public Law No. 111-148, Section 3502, 124 Stat. 119,124

Medical CRS Report. (2018). The Federal Assistance Percentage (FMAP). Medical CRS Report.

Menard, S. (1997). Applied logistic regression analysis (2nd ed., Vol. 106, Paper series on quantitative applications in the social sciences). Thousand Oaks, CA: Sage Publication.

Mesch,M.P.(1999). The effects of Hospital Closure on Mental Health Workers. Journal of Behavioral Health Services and Research, 305-317.

Mitchell JM, G. D. (2008). Health supervision visits among SSI eligible children in the D.C. Medical program.

Mosser, G., MD, Karp, M., MBA, \& Rabson, B. G., MPH. (2006, August 1). Regional coalitions for healthcare improvement: Definition, lessons, and prospects. Retrieved January, 2015, from http://www.improvingchroniccare.org/downloads/nrhi.pdf

Munoz E, S.R. (1986). Source of Admission and Cost; Public Hospitals Face Financial Risk. American Journal of Public Health, 696-697.

Muss, L.G. (1995). Community Perceptions of Rural Hospital Closure. Journal of Community Health, 65-73.

Musumeci, M. (2012, August 1). A Guide to the Supreme Court's Decision on the ACA's Medicaid Expansion. Retrieved June, 2016, from https://www.kff.org/healthreform/issue-brief/a-guide-to-the-supreme-courtsdecision/

National Conference of State Legislatures. (2011, March). The Affordable Care Act: A brief summary. Retrieved October, 2018, from http://www.ncsl.org/research/health/the-affordable-care-act-brief-summary.aspx

National Quality Forum. (2011). Prevention EM Phase 1 Screening and Immunization Measures.

ObamaCareFacts.com. (2014, August 8). State health insurance exchange: State run exchanges. Retrieved February, 2017, from https://obamacarefacts.com/statehealth-insurance-exchange/

Patel, K., \& Rushefsky, M. E. (2006). Health care politics and policy in America (3rd ed.). Armonk, N.Y.:

Patient Protection and Affordable Care Act, Public Law No. 111-148, Section 3502, 124 Stat. 119,124.

Piore, P.a. (1977). Public expenditures and private control? Health Care Dilemmas in New York City. Milbank Memorial Fund Quarterly, pp.79-116.

Probst, S.H. (1990). Economic Impact of Hospital Closure on Small Rural Counties, 1984 to 1988. Journal of Rural Health, 375-390.

Robert Wood Johnson Foundation University of Wisconsin Population Health Institute. (2011). County Health Ranking.

Rosenblatt RA, A.C. (2006). Shortages of medical personnel at Community Health Centers: Implications for Planned Expansion. JAMA.

Rothman, D. J. (1993). A century of failure: Health care reform in America. Journal of Health Politics, Policy and Law, 18(2).

Ross, M., \& Hayes, C. (1986). Consolidated Omnibus Budget Reconciliation Act of 1985 (Vol. 49, No. 8, Publication). Retrieved August, 2013, from https://www.ssa.gov/policy/docs/ssb/v49n8/v49n8p22.pdf

Schuster CR,M.J.(2007). Partially capitated managed care versus FFS for Special Needs Children. Health Care Finance Review, 109-123.

Shepard. (1983). Estimating the Effects of Hospital Closure on Area wide Inpatient Hospital Cost: A Preliminary Model and Application. Health Services Research.

Smith, J. C., \& Medalia, C. (2014, September). Health Insurance Coverage in the United States: 2013 (United States, United States Census Bureau). Retrieved August, 2017, from 
https://www.nber.org/cps/hi/2014redesign/p60-250.pdf

Sohn, H. (2016). Racial and ethnic disparities in health insurance coverage: Dynamics of gaining and losing coverage over the life-course. Population Research and Policy Review, 36(2), 181-201. doi:10.1007/s11113-016-9416-y

The Lewin Group. (2006). Medicaid upper payment Limit Policies: Overcoming a Barrier to Managed Care Expansion. Medicaid Health Plans of America.

The Johns Hopkins University, \& Weiner, J. P., DrPH. (2008). Health policy and the delivery of health care: Introduction and private health plan case study. Lecture. Retrieved August, 2014, from http://ocw.jhsph.edu/courses/IntroHealthPolicy/PDFs/IHP lec7 weiner.pdf

Trust for America's Health. (2009). Shortchanging America's Health: A State by State Look at How Federal Public Health Dollars are Spent.

United Health Foundation. (2010). America's Health Rankings. United Health Foundation

United States Congress UMTALA Act. (1986). 42 U S C 1395dd EMTALA ACT.

Urban Institute and Kaiser Commission on Medicaid and the Uninsured. (2009/2010). Uninsured Estimates based on Census Bureau Population Surveys.

Urban Institute. (2010). Kaiser Commission on Medicaid and the Uninsured. Urban Institute.

Waitzkin (2005). The History and Contradictions of the Health Care Safety Net.

Whiteis D, S.J. (1978). The Proprietarization of Health Care and the Underdevelopment of the Public Sector. International Journal of Health Services. 47-64.

Williams, H.P. (1992). Profits, Community Role and Hospital Closure: An Urban and Rural Analysis. Medical Care, 174

Williams, W. (1980). Review of The Implementation Perspective a Guide for Managing Social Service Delivery Programs. University of California Press.

Williams, D. R., Mohammed, S.A., Leavell, J., \& Collins, C. (2010). Race, socioeconomic status, and health: complexities, ongoing challenges, and research opportunities. The Biology of Disadvantage: Socioeconomic Status and Health. Annals of the New York Academy of Science. , 1186, 69-101. doi: 10.1111/j.17496632.2009.05339.x 